\title{
Estudios críticos en discapacidad: aportes epistemológicos de un campo plural
}

\section{Critical disability studies: epistemological contributions from a plural field}

Beatriz Revuelta (beatriz.revuelta@ucentral.cl) Facultad de Educación y Ciencias Sociales, Universidad Central de Chile (Santiago, Chile) https://orcid.org/0000-0002-1550-4960

Raynier Hernández (rhernandeza@udla.cl) Facultad de Salud y Ciencias Sociales, Universidad de Las Américas (Santiago, Chile) https://orcid.org/0000-0002-8205-0081

\begin{abstract}
Critical disability studies have become over the last three decades an interdisciplinary academic field that introduces a plural and diverse path to understanding disability. These studies focus on a series of theoretical developments that privilege cultural, discursive, and relational understandings of disability, connecting the aspirations of people with disabilities with the agendas of feminist, queer, postcolonial, poststructuralism and posthuman studies. This work aims to outline the epistemological contributions of these studies through the identification of theoretical axes that have been consolidated: power, production of bodies and internalized oppression; intersectionality and multiple identities; and posthuman assemblages. The contribution of these axes lies on the one hand, in the departure from the disability-impediment binary characteristic of the more conventional social studies of disability, which allows us to understand the variability of experiences of disability, and, on the other hand, it makes disability concept strategic to explain the ways in which subjects are produced in current societies.
\end{abstract}

Key words: critical studies, disability, bodies, oppression, intersectionality, subjects.

\section{Resumen}

Los estudios críticos en discapacidad se han convertido durante las últimas tres décadas en un campo académico interdisciplinario que introduce un camino plural y diverso para la comprensión de la discapacidad. Estos estudios se enfocan en una serie de desarrollos teóricos que privilegian las comprensiones culturales, discursivas y relacionales de la discapacidad, conectando las aspiraciones de las personas con discapacidad con las agendas de los estudios feministas, queer, poscoloniales, postestructuralistas y posthumanos. Este trabajo tiene como objetivo esbozar los aportes epistemológicos de estos estudios a través de la identificación de ejes teóricos que se han ido consolidando: poder, producción de los cuerpos y opresión internalizada; interseccionalidad e identidades múltiples; y ensamblajes posthumanos. La contribución de estos ejes radica, por un lado, en la salida del binarismo discapacidad-impedimento propio de los estudios sociales de la discapacidad más convencionales, lo que permite comprender la variabilidad de experiencias de la discapacidad, y, por otro lado, hace entender el concepto discapacidad como estratégico para explicar las formas en las que se producen los sujetos en las sociedades actuales.

Palabras clave: estudios críticos, discapacidad, cuerpos, opresión, interseccionalidad, sujetos. 


\section{Introducción}

Desde 1970 se comenzó a pensar la discapacidad como una construcción social y a las personas con discapacidad como un grupo históricamente oprimido y políticamente no reconocido bajo la lógica de los derechos humanos. Desde esta época, se buscó expandir la comprensión medicalizada de la discapacidad que la consideraba una forma negativa de ser que necesitaba una solución médica, mediante la introducción de un modelo social de la discapacidad que comenzó en el Reino Unido. El modelo social se basó directamente en la teoría crítica, "examinando al igual que ella las interrelaciones entre el sistema capitalista de producción, clase y discapacidad, y defendiendo una perspectiva emancipadora dentro de los estudios de discapacidad" (Meekosha y Shuttleworth 2009:50). Impulsó la definición de la discapacidad no como un defecto físico inherente a los cuerpos, sino como una forma de comprender estructuras opresivas. De este modo, la discapacidad se convirtió en un sistema de representación más que un problema médico o una desgracia personal asociada a un cuerpo.

Este movimiento, articulado en el manifiesto de la Unión de Discapacitados Físicos, "buscaba unir a las personas con discapacidad como un grupo común, y trasladar la causa de la desventaja [...] a la sociedad en general y las estructuras físicas y sociales construidas que incapacitan a las personas con discapacidad" (Ellis, Garland-Thomson y Kent 2019:3). Esta epistemología liberadora separó el deterioro físico, que puede ser invariable, de la discapacidad como una restricción impuesta socialmente. De este modo, se comienza a distinguir la deficiencia como "los estados o condiciones corporales que se consideran deterioros físicos, y la discapacidad como el proceso social que da significado y produce ciertas consecuencias para esas personas" (Garland-Thomson 2011:591).

Con este cambio se introdujo el término de opresión. Referirse a la discapacidad como opresión significó reconocer y visibilizar la posición de inferioridad de las personas con discapacidad respecto de otros en la sociedad, y también dar cuenta de que esa posición estaba relacionada con ideologías que la justificaban y perpetuaban. Significó dar cuenta también de que "dichas desventajas y las ideologías que las sostenían no eran ni naturales ni inevitables" (Abberley 2008:37).

Este enfoque para explicar la causa fundamental de las exclusiones sociales continúa siendo la base fundamental de los estudios sociales sobre discapacidad que se desarrollan en la actualidad. Sin embargo, y a pesar del reconocimiento de los aportes de este modelo para posicionar la discapacidad como un asunto social, y no como uno netamente médico, comenzaron a surgir varias críticas a fines de la década de 1990. Las dos principales críticas a este modelo han sido: el pensamiento en binarios discapacidad-deficiencia física que como separación teórico-política no permitía una fácil articulación y explicación de la complejidad inmersa en las experiencias de la discapacidad, y el "poner demasiado énfasis en las barreras estructurales, ignorando así las dimensiones culturales, corporales y vivenciales de la discapacidad" (Reeve 2002:495).

Si bien la dicotomía entre discapacidad y deficiencia fue políticamente potente como reconocimiento del carácter social de la discapacidad, por otro lado, impidió una comprensión clara respecto de la realidad de personas cuya constitución como sujetos siempre se jugaba en la relación entre cierta condición corporal y un contexto específico. Estos casos evidenciaron que hablar de discapacidad tampoco era solamente hacer referencia a una opresión externa o estructural. Así, "lo que cuenta como discapacidad varía en un amplio espectro de condiciones físicas, motoras, 
mentales, sensoriales, conductuales, médicas y de apariencia que restringen la función, limitan la participación y se entienden como formas estigmatizadas de inferioridad" (Ellis, Garland-Thomson y Kent 2019:13).

Derivado de estas críticas al modelo social y de una imbricación sostenida entre contribuciones teóricas interdisciplinares directamente relacionadas con movimientos emancipatorios, se ha abierto paso en las últimas tres décadas un conjunto de estudios que se han denominado Estudios Críticos en Discapacidad. Un campo académico que introduce un camino plural y diverso que está menos centrado en el imperativo materialista relativo a cómo las estructuras materiales restringen y oprimen ciertos cuerpos, y privilegia las comprensiones culturales, discursivas y relacionales. De este modo, se han caracterizado por "conectar las aspiraciones de las personas con discapacidad con las agendas transformadoras de los estudios feministas, queer, postestructuralistas, poscoloniales y posthumanos" (Goodley, Hughes y Davis 2012:174). También se ha reconocido que representan un esfuerzo por "desafiar el dominio de las preocupaciones del Norte Global en la producción de conocimientos en torno a la discapacidad" (Mallett y Runswick-Cole 2015:16).

El presente trabajo tiene como objetivo esbozar los aportes epistemológicos de estos estudios críticos a través de la identificación de ejes teóricos de comprensión que se han ido consolidando: poder, producción de los cuerpos y opresión internalizada; interseccionalidad e identidades múltiples; y ensamblajes posthumanos. La contribución de estas áreas a pensar la discapacidad radica, por un lado, en la salida del binarismo discapacidad-deficiencia física propia de los estudios sociales de la discapacidad más convencionales, y por otro lado, permite comprender la discapacidad como un concepto que no es solamente relativo a cuerpos con ciertas "condiciones" en interacciones múltiples, sino que es estratégico para explicar las formas en las que se producen a sí mismos y son producidos los sujetos en las sociedades actuales.

\section{Los estudios críticos en discapacidad: tres ejes de discusión teórica}

La diferencia de los estudios críticos en discapacidad radica en la incorporación de una amplia gama de enfoques epistemológicos divergentes. De acuerdo con Garland-Thomson, el pensamiento binario promovido por los estudios sociales sobre discapacidad en sus inicios "no podía capturar la rica combinación de estados corporales que constituyen un enfoque más matizado a la cuestión de la deficiencia. Esta [la deficiencia] es un factor general que abarca diferencias físicas congénitas y adquiridas, enfermedades mentales y retraso, enfermedades crónicas y agudas, enfermedades mortales y progresivas, lesiones temporales y permanentes y una amplia gama de características corporales consideradas desfigurantes, como cicatrices, marcas de nacimiento, proporciones inusuales, u obesidad. Las deficiencias físicas que hacen que alguien tenga discapacidad casi nunca son absolutas o estáticas; son condiciones dinámicas, contingentes, afectadas por muchos factores externos y generalmente fluctúan con el tiempo" (Garland-Thomson 2017:13).

En este sentido, los estudios críticos abrieron una puerta importante a la comprensión de la variabilidad humana. Esta puerta incorporó, además del reconocimiento de una amplia gama de deficiencias, impedimentos o limitaciones funcionales juzgadas socialmente, "preocupaciones interseccionales, como las de etnia, edad, clase, sexualidad, y género, que impactan en la experiencia de la discapacidad" (Shildrick 2012:33). Los estudios críticos introducen así un conjunto de reflexiones transversales que permiten cuestionar el modo en que se construye sociedad. Desde 
la discapacidad, es posible comprender la cristalización de mecanismos reguladores que permiten desechar la variabilidad que "incomoda" a la verdad que normaliza y estandariza los cuerpos. La forma en que las sociedades dividen los cuerpos y los etiquetan, excluyen y manejan las emociones a través de ciertos dispositivos y tecnologías, resulta muy relevante para pensar la discapacidad y las realidades de exclusión y vulnerabilidad en donde se entrecruza el género, la raza, la clase y la discapacidad.

Las definiciones y verdades dominantes que circulan "determinan la elección y participación en la vida cívica de ciertos grupos, determina qué constituye el ser hombres y mujeres racionales y tener acceso a ciertos derechos y garantías, y también determina quién debe tener derecho a ser parte de la sociedad y quién no" (Meekosha y Shuttleworth 2009:65). Como veremos en el desarrollo de los diferentes ejes teóricos, las reflexiones que derivan de estos aportes enuncian de forma muy clara el carácter social de la discapacidad, y las maneras en que la discapacidad se convierte en un concepto muy relevante que, unido a otros, explica las formas en que funciona el sistema social con la diferencia.

Goodley y Lawthom han definido las características y aportes de los estudios críticos en discapacidad de la siguiente manera:

"-Reconocen la importancia de analizar la discapacidad a través del materialismo y es respetuoso con los componentes básicos de los estudios sobre discapacidad, especialmente el modelo social de discapacidad.

-Reconocen que nuestros tiempos contemporáneos son complejos porque están marcados por la austeridad, una brecha cada vez mayor entre ricos y pobres, la globalización de los principios rectores del capitalismo tardío y, por lo tanto, requieren teorías sociales sofisticadas que puedan cuestionar estos procesos.

-Permanecen atentos a los contextos económicos globales, nacionales y locales y su impacto en las personas con discapacidad.

-Adoptan una posición de relativismo cultural mientras buscan decir algunas cosas sobre la naturaleza global de la discapacidad.

-Reconocen la importancia de la constitución del yo en relación con los demás (y, por lo tanto, siempre está en sintonía con las cualidades relacionales de la discapacidad).

-Adoptan la práctica de la criticidad para ser crítico con todo tipo de estudios de discapacidad (incluidos los estudios críticos de discapacidad).

-Tienen en cuenta la opinión de que cualquier análisis de la discapacidad no debe excluir la consideración de otras formas de activismo político" (Goodley y Lawthom 2019:234).

La propuesta de ejes teóricos que se presenta en las próximas páginas deriva de una sistematización de los aportes realizados por estos estudios, y tiene su base en las propuestas de otros teóricos del tema que han intentado sistematizar en los últimos años los alcances del campo. Si bien otras sistematizaciones han relevado los aportes de un país a este campo o han intentado reconocer los aportes a partir de la focalización en grupos particulares, en este trabajo buscamos encontrar los marcos más generales que son "caja de herramientas" para analizar las realidades heterogéneas que constituyen a los sujetos con discapacidad.

Como es un campo en movimiento, resulta fundamental el reconocimiento de ciertos anclajes teóricos más globales que permitan impulsar la investigación social en discapacidad desde estos 
nuevos lentes. Los ejes que se presentan no están ceñidos o no responden solamente a disciplinas o posturas teóricas específicas, si no que tributan a ellos diferentes enfoques y teorías que se filtran continuamente, dialogan y coproducen, para generar comprensiones relacionales, situadas y diversas sobre la discapacidad y las personas con discapacidad. Se propone discutir en torno a los siguientes ejes:

\begin{tabular}{|c|c|}
\hline \multicolumn{2}{|c|}{ Estudios Críticos en Discapacidad } \\
\hline Ejes de producción teórica & Líneas \\
\hline $\begin{array}{l}\text { Poder, producción de los cuerpos y } \\
\text { opresión internalizada }\end{array}$ & $\begin{array}{l}\text { Biopoder y producción del sujeto } \\
\text { Sujetos inscritos con la diferencia (estigma) } \\
\text { La ideología de la normalidad y el capacitismo } \\
\text { El resultado de los dispositivos: la opresión } \\
\text { internalizada }\end{array}$ \\
\hline $\begin{array}{l}\text { Interseccionalidad e identidades } \\
\text { múltiples }\end{array}$ & $\begin{array}{l}\text { Feminismo y discapacidad } \\
\text { Sexo, sexualidad y discapacidad } \\
\text { Discapacidad racializada }\end{array}$ \\
\hline Ensamblajes posthumanos & $\begin{array}{l}\text { Humanos y no humanos se constituyen en la } \\
\text { superación de la "normalidad" } \\
\text { Cyborg }\end{array}$ \\
\hline
\end{tabular}

\section{Poder, producción de los cuerpos y opresión internalizada}

La historia de la discapacidad a lo largo de la modernidad ha sido una historia de patologización y supervisión, de control de los cuerpos y aislamiento. Si se analiza la realidad actual de las personas con discapacidad, se podría decir que "continúa la exclusión discursiva y material coexistiendo con programas de cambio concertados, y a menudo efectivos, que avanzan hacia la integración formal de las personas con discapacidad en los derechos, obligaciones y expectativas estándar de la ciudadanía normativa. Sin embargo, ser percibido como encarnado de manera diferente es ocupar un lugar definido como excepcional, en lugar de simplemente ser parte de una multiplicidad de posibilidades. A pesar de las formas infinitamente diferenciales de encarnación humana, el discurso dominante continúa marcando a algunas personas, pero no a otras, como inherentemente excesivas a los límites normativos" (Shildrick 2012:31). En este sentido, la diferencia sigue siendo devaluada por aquellos que pueden alinearse a los estándares de "normalidad" que persisten en el imaginario social. En este apartado se desarrollan los aportes de varios enfoques críticos para pensar las diversas realidades opresivas. Lo interesante de este primer eje es que se separa de los estudios sociales de la discapacidad en su etapa inicial, para privilegiar análisis asociados al manejo de la subjetividad y a la producción del yo, sin perder de vista la importancia de las estructuras opresivas en la legitimación de las diferencias.

Biopoder y producción del sujeto. Los aportes de Michael Foucault a la comprensión de las prácticas médicas, administrativas y legales que legitimaron los procesos de patologización de los cuerpos "diferentes" son fundamentales. Si bien es posible encontrar algunas críticas a la teoría foucaultiana respecto a la capacidad de agencia disminuida o nula de los sujetos en la era del biopoder, sin dudas sus análisis en torno al poder, la sociedad carcelaria, la gubernamentalidad, el gobierno y los dispositivos que clasifican, normalizan, manejan y controlan sujetos corporales "anómalos", han hecho posible entender cómo se han producido las verdades dominantes en torno a la discapacidad 
y finalmente como el "Yo" con discapacidad se ha configurado a sí mismo en este contexto: "Lo que distingue el punto de vista foucaultiano sobre la deficiencia y la discapacidad de la perspectiva materialista dominante de los estudios sociales iniciales es el poderoso papel que el primero asigna al lenguaje y al simbolismo en la construcción de identidades" (Hughes 2005:81) y la relación causal que es posible encontrar entre la producción de la deficiencia (discurso médico) y la discapacidad. Su concepción del poder y su vínculo con el cuerpo ofrece una manera de explicar las prácticas que han separado, institucionalizado y normalizado de diversas maneras a las personas con discapacidad: "Se podría argumentar que la discapacidad es un producto del biopoder modernista, es decir, un efecto del tratamiento médico de las personas con deficiencias, [...] es un producto del discurso médico-asistencial" (Hughes 2005:82).

En este sentido, el enfoque del poder propio del pensamiento foucaultiano difiere de la tradición marxista que dominó hasta la década de 1990 los estudios sociales de la discapacidad. "Esta última promueve una visión del poder que lo interpreta como unitario, centralizado y principalmente coercitivo" (Hughes 2005:83). Las discusiones de Foucault relativas al advenimiento de la noción de "normalidad" desde el discurso médico y las políticas de encierro y aislamiento que tal discurso generó, son claves para comprender una nueva forma de poder centrada en los cuerpos, en el disciplinamiento, corrección y manejo de las rutinas: el biopoder. Las operaciones de esta forma de poder establecieron finalmente la idea de que la capacidad tiene su opuesto en la discapacidad, y que ambos conceptos se sustentan desde concepciones biológicas sobre el cuerpo promedio "normal" versus el que no lo es. En este sentido, la capacidad existe a través de la discapacidad y viceversa.

El biopoder tuvo su expresión más clara en el aislamiento, pero también en el desarrollo de un conjunto de tecnologías que permitieran tratar al "cuerpo anómalo". Estas "tecnologías de la normalización no fueron meras respuestas inocuas o incluso benevolentes a estas anomalías en el cuerpo social. Por el contrario, fueron centrales para la creación, identificación, clasificación y control de tales anomalías; es decir, contribuyeron a la constitución de la percepción de anomalías (como deterioro) y operaron como mecanismos a través de los cuales algunos sujetos se podían ‘justificadamente' dividir de los demás” (Tremain 2020:55).

El concepto de gobierno como el de gubernamentalidad también aporta a la comprensión de las realidades de las personas con discapacidad. La gubernamentalidad implica una conexión entre técnicas de gobierno de los otros y las de gobierno de sí mismo dentro de un dispositivo histórico. Nos permite pensar los efectos propios de determinada tecnología de poder, no solo en relación con la "conducción de las conductas de los otros" (Foucault 2007:360), sino vinculando ésta con las prácticas que un individuo es llevado a realizar sobre sí. Finalmente, la construcción del "yo" con discapacidad va a estar completamente coactada por un sinnúmero de verdades dominantes basadas en las diferencias físicas o conductuales como "excepcionales" y manejadas por profesionales de la medicina que tienen como función principal diagnosticar para rehabilitar y "normalizar" lo más posible las "desviaciones".

El trabajo de Foucault arroja luz además sobre el conjunto de prácticas históricamente contingentes que restringen las acciones de las personas con discapacidad. En este enfoque, "las deficiencias son fabricaciones médicas que constituyen la discapacidad como una posición de sujeto 'natural'. Desde 
una perspectiva foucaultiana, la normalización del juicio constituye a la deficiencia como un déficit de integridad corporal y, simultáneamente, como una posición social inválida" (Hughes 2005:82).

Sujetos inscritos con la diferencia (estigma). Otra teoría que fue relevada como fundamental, incluso dentro de los primeros estudios sociales sobre discapacidad, es la iniciada por Erving Goffman, y que refiere a aquellos mecanismos de clasificación que marcan a ciertos sujetos. La teoría del estigma aportada por este autor proporciona un vocabulario y brinda un aparato teórico útil para ubicar la discapacidad en contextos sociales específicos. "Los individuos son marcables debido a rasgos particulares, y los marcadores son aquellos que interpretan ciertos rasgos como desviados. La teoría del estigma, por lo tanto, proporciona un medio para rastrear con precisión la producción de minorías u 'otros' culturales. En resumen, estigmatizar describe las distinciones entre las personas, sus rasgos físicos, lo que se les hace, quién lo hace y qué significa" (Garland-Thomson 2017:31).

El estigma constituye así un dispositivo de opresión, "un mecanismo de poder que hace posible que unos grupos estigmatizados queden desempoderados" (Scior y Werner 2019:5) y tributa directamente a la comprensión del "grado de reconocimiento" que tendrá un sujeto (Revuelta y Hernández 2019:344). La teoría del estigma "es útil porque desenreda los procesos que construyen tanto lo normativo como lo desviado y revela los paralelismos entre todas las formas de opresión cultural y, al mismo tiempo, permite que las identidades devaluadas específicas permanezcan a la vista, esencialmente resitúa el 'problema' de la deficiencia del cuerpo de la persona con discapacidad al marco social de ese cuerpo" (Garland-Thomson 2017:31).

Finalmente, la teoría del estigma recuerda que la discapacidad es una construcción social, y que los problemas que se enfrentan derivados de la discapacidad, la etnia, la raza, la clase, la homosexualidad o el género, son incongruencias, actitudes negativas, tergiversaciones y prácticas institucionales que resultan del proceso de estigmatizar, del proceso de clasificar a otros bajo ciertos atributos indeseados. El estigma tiene su base y deriva de las intersecciones que constituyen a los "otros" en las diferentes sociedades.

La ideología de la normalidad y el capacitismo. El capacitismo es una epistemología que enmarca la formación de la identidad de un individuo en las actuales sociedades. Prestar atención a lo que implica esta forma de organizar el mundo para las personas con discapacidad resulta clave para comprender los procesos sistemáticos de opresión en expresiones discriminatorias. "Para los regímenes de capacitismo son fundamentales dos elementos centrales, a saber, la noción de lo normativo y la aplicación de una división constitucional entre la humanidad naturalizada perfeccionada y el híbrido aberrante, impensable, cuasi-humano, y por lo tanto no humano. Esta constitución proporciona el diseño, el plano para la escala y el marcado de los cuerpos y el orden de sus términos de relación" (Campbell 2009:6).

El capacitismo da cuenta de las "prácticas sofocantes asociadas a una sociedad contemporánea que busca cada vez más promover la especie típica del ciudadano individual: un ciudadano que está listo y es capaz de trabajar, contribuir productivamente, un fenómeno atomista acotado y aislado de otros, capaz, maleable y dócil" (Goodley y Lawthom 2019:325). De este modo, a las personas con discapacidad se les prohíbe la ciudadanía plena porque sus cuerpos no se ajustan a "las convenciones legales [...] basadas en suposiciones de que los cuerpos parecen y funcionan de cierta 
manera" (Garland-Thomson 2017:46). La ideología de la normalidad que incluye la noción de capacidad condiciona de forma central las vidas y experiencias de las personas con discapacidad. Establece una clara distinción entre el cuerpo "normal" del que no lo es, y tiene repercusiones importantes en los marcos del reconocimiento e implicaciones prácticas relacionadas con la integración y acceso a oportunidades. La lógica capacitista propia de las sociedades capitalistas actuales denigra sujetos cuyas condiciones corporales, conductuales, o conflictivas con las representaciones sociales dominantes, no deben mostrarse o acceder a espacios de reconocimiento mayor. En el caso de las personas con discapacidad, el capacitismo se evidencia de forma muy clara en el acceso al empleo, en el acceso a la educación o a la configuración de espacios sociales accesibles.

Salvando las diferencias individuales, podría decirse que la experiencia de la discapacidad representa un trauma comunitario o colectivo donde los legados del capacitismo perduran y se reproducen. "Las historias de fracasos, las representaciones negativas y la ausencia de modelos celebradores saturan colectivamente la vida de las personas con discapacidad" (Campbell 2009:22).

El resultado de los dispositivos: la opresión internalizada. Derivado entonces de los procesos históricos de violencia y opresión sobre los cuerpos, de la posición cada vez más enquistada respecto de los otros "raros" sobre los "normales", han surgido diversos análisis relativos a la performatividad del sujeto, que dan cuenta de que la opresión llega a formar parte de su constitución y define sus prácticas. La opresión internalizada puede describirse como la incorporación y aceptación por parte de un grupo oprimido de los prejuicios hacia ellos dentro de la sociedad dominante. Es a su vez, "un mecanismo [...] para perpetuar la dominación no solo mediante el control externo, sino también mediante la construcción de la sumisión en las mentes de los grupos oprimidos" (Reeve 2002:495). Esta definición se encuentra en estrecha relación con las discusiones sostenidas con anterioridad respecto del biopoder, el estigma, el capacitismo y cómo influyen en la constitución del "yo". Para oprimir se necesita contar con sujetos disciplinados que han producido una versión de sí ajustada a las necesidades del sistema. Las personas con discapacidad internalizan los prejuicios y estereotipos que provee la "normalidad"; y esto hace posible gobernar los cuerpos y controlarlos a partir de un régimen de verdad dominante que los ha etiquetado, excluido y situado en un espacio específico de reconocimiento en cada sociedad.

La opresión se expresa de manera clara en los afectos y las emociones derivados de las experiencias de las personas con discapacidad. Una crítica a los primeros estudios sobre discapacidad es que prestaron más atención a las experiencias públicas de opresión, como las barreras sociales, a expensas de las experiencias más personales de opresión que operan a nivel emocional. "Estas dimensiones psicoemocionales de la discapacidad, que afectan a las personas incluyen ser lastimados por las reacciones de otras, sentirse inútiles y poco atractivos, y tienen sus raíces en las actitudes negativas y prejuicios sobre las personas con discapacidad dentro de la sociedad" (Reeve 2002:495).

De este modo, es posible identificar también un proceso de auto estigma. "El auto estigma, como el estigma público, comprende estereotipos, prejuicios y discriminación. Los estereotipos como 'soy una persona débil' o 'soy incapaz' son respaldados por el individuo y conducen al auto prejuicio en forma de respuestas emocionales negativas. Esto, a su vez, puede conducir a la auto discriminación a través de respuestas de comportamiento como no buscar oportunidades de empleo o evitar las 
relaciones sociales. Este proceso implica que un sujeto ha aceptado acríticamente un estereotipo negativo porque cree que aplica a él. El auto estigma puede ocurrir en ausencia de experiencias reales de discriminación, debido a la anticipación o el miedo al rechazo o la devaluación" (Sheehan y Ali 2019:92). Da cuenta de procesos de opresión que han sido incorporados con anterioridad por medio de las experiencias de interacción con otros.

En relación con ello, desde las teorías que se encargan de pensar las consecuencias del colonialismo en los "dominados", la opresión internalizada ha sido un concepto central. Tomando como base a Franz Fanon, se han generado análisis relativos a la deshumanización y cómo los colonizados internalizaron su opresión. Las personas con discapacidad experimentan alienación de sus propios cuerpos, de su sexualidad y de otros en la sociedad. A consideración de Charlton: "su evolución de la conciencia está informada en su mayor parte por vidas con privaciones económicas y sociales en las que se les dice todos los días, de una forma u otra, que son patéticos, grotescos y, lo que es más significativo, inferiores. Este mensaje se ve reforzado por una variedad de instituciones sociales" (Charlton 2004:70). Esto implica la interiorización de una "falsa verdad" que tiene consecuencias psicoemocionales muy significativas.

En relación con el capacitismo desarrollado en el punto anterior, se reconoce que es uno de los procesos que internalizan con fuerza las personas con discapacidad. Para llegar a la norma se requiere que el sujeto se apropie de una identidad distinta. Así, "las personas con discapacidad a menudo se sienten obligadas a fabricar 'quiénes' son, y a adoptar aspectos que son adicionales a ellos mismos" (Campbell 2009:26). Esta obligación pasa por asimilarse lo más posible a la norma capacitista y esconder, obviar o dejar de compartir con los otros las formas en que experimentan su vida.

\section{Interseccionalidad e identidades múltiples}

El eje interseccionalidad e identidades múltiples tiene su base en las variadas intersecciones que complejizan la producción de los sujetos con discapacidad. La conjunción de la discapacidad, el género, la raza, la sexualidad o la clase, en interacción con contextos culturales, económicos y políticos específicos hace que se configuren vivencias muy diferenciadas y únicas de vivir la discapacidad. Este eje aporta una mirada multidimensional de las opresiones, que obliga a pensar sobre fenómenos más amplios relativos a los horizontes morales, aquellos valores que son deseablemente comunes a todos y cómo corren en las sociedades actuales empeñadas en patologizar, rehabilitar y normalizar la diferencia. Estas categorías "son todas construcciones sociales de exclusión y procesos de nombrar y clasificar quién constituye y quién no constituye un ciudadano pleno" (Meekosha 2006:170).

Feminismo y discapacidad. Dentro de los estudios críticos, la corriente feminista para pensar la discapacidad y las mujeres con discapacidad ha sido central. Esto se debe a los "muchos paralelos que existen entre los significados sociales atribuidos a los cuerpos femeninos y a los cuerpos con discapacidad. Ambos han cargado con atributos de inferioridad y desviación, ambos han sido excluidos de poder participar en el ámbito público y en la vida económica. Ambos han sido definidos en oposición a la norma que se ha considerado como algo físicamente superior" (Garland-Thomson 2017:19). 
Los estudios feministas han demostrado que las relaciones sociales que constituyen la discapacidad se articulan con las que constituyen las relaciones de género. La tarea de examinar la interacción de la discapacidad y el género se volvió más desafiante a medida que las perspectivas feministas sobre la naturaleza de las relaciones de género en sí mismas "se fragmentaron y sintonizaron con múltiples diferencias desde la década de 1980 en adelante" (Thomas 2004:4). A consideración de Judith Butler, el género no siempre se constituye de forma coherente o consistente en contextos históricos distintos, porque además "se entrecruza con modalidades raciales, de clase, étnicas, sexuales y regionales, de identidades discursivamente constituidas. Así, es imposible separar el género de las intersecciones políticas y culturales en las que constantemente se produce y se mantiene" (Butler 2007:49). Del mismo modo pasa cuando se piensa en la discapacidad, surge desde una multiplicidad que escapa a una relación binaria y naturalizada con un cuerpo, sino que es posible su comprensión como una construcción situada contextual y culturalmente y discontinua, sin formas predefinidas, sin espacios de cierre o encuadres estancos. Entonces la discapacidad une a un grupo heterogéneo cuya única característica en común es que se consideran fuera de la "norma".

Lo que aporta el feminismo en términos generales es a pensar la discapacidad como una categoría que resiste o desafía, del mismo modo que el género, las relaciones sociales existentes. "Ambas se resisten a las interpretaciones de ciertas configuraciones corporales y funcionan como desviadas de la norma; ambas cuestionan las formas en que las diferencias están investidas de significado social; ambos examinan la aplicación de normas universalizantes; ambas interrogan la política de la apariencia" (Garland-Thomson 2017:22). En este sentido, Garland-Thomson propone una visión universalizadora de la discapacidad, mostrando cómo el concepto de discapacidad informa a ideologías nacionales e identidades varias. Entiende la discapacidad como un concepto que "puede utilizarse para desafiar la suposición persistente de que es una condición evidente de insuficiencia física y desgracia privada que concierne solo a una minoría limitada" (Garland-Thomson 2017:22).

El feminismo ha ayudado también a visibilizar las realidades diferenciadas de las mujeres con discapacidad en torno a la construcción de su identidad. Desde los estudios feministas se censura la cosificación sexual de las mujeres, sin embargo, las mujeres con discapacidad a menudo se encuentran con cosificación asexual, porque existe la suposición de que la sexualidad es inapropiada para las personas con discapacidad. "El juicio de que el cuerpo de la mujer con discapacidad es asexual y poco femenino, genera una invisibilidad social y encarcelamiento de la feminidad que puede impulsar a las mujeres con discapacidad a adquirir la identidad femenina que la cultura les niega, a contrapelo de la crítica feminista relacionada con la cosificación de los cuerpos. El sentirse más alineada a la 'norma' se vuelve problemático y en general ha sido poco tematizado" (GarlandThomson 2017:25).

Otra discusión teórica interesante relativa a las experiencias de las mujeres con discapacidad es aquella que polemiza en torno a la creencia de los cuerpos como no aptos para la maternidad o de las mujeres con discapacidad como objetos infantilizados que motivan la caridad y solidaridad ajena. "Cuando la maternidad se considera a menudo obligatoria para las mujeres, a las mujeres con discapacidad se les niega o desalienta el papel reproductivo que algunas pensadoras feministas encuentran opresivo" (Garland-Thomson 2017:26).

Por otro lado, el rol infantilizado propio de las concepciones medicalizadas de la discapacidad hace que la persona pierda el derecho a decidir respecto de su vida y su cuerpo. Es importante significar 
que este hecho tiende a confundirse con la idea de que las personas con discapacidad deben recibir y son sujetos de cuidados y atención permanente, de alguien que decide por ellos. Ocupar los cuidados como contrarios a la capacidad de decisión y a la independencia y la autonomía, es una forma limitada de observarlo y de considerarlo en el ámbito relacional y práctico de la discapacidad. Muchas personas con discapacidad requieren de los cuidados para desarrollar una vida digna, requieren de apoyos permanentes para la realización de sus actividades cotidianas. Y ello no los vuelve pasivos, o con poca capacidad para decidir, no los infantiliza, sino que les posibilita justamente "ser parte de", "participar de la vida de manera digna".

En este sentido, la forma en que se construye una identidad diferenciada para las mujeres con discapacidad es potente para relevar fenómenos que están deconstruyéndose desde el feminismo, pero que constituyen puntos que aún no se desarrollan con suficiencia para estos grupos que distan de la "normalidad" y no "disfrutan-sufren" de la asignación de los roles tradicionales de madre, jefa de hogar o cuidadora, o de tener un cuerpo ajustado a la moda del momento. "En una sociedad en la que la apariencia es el principal índice de valor para las mujeres (y cada vez más para los hombres), las prácticas de embellecimiento normalizan el cuerpo femenino y las discapacidades lo 'anormalizan'. La feminización aumenta el capital cultural de la mujer; la discapacidad lo reduce" (Garland-Thomson 2017:28). De la misma manera se produce una configuración de las identidades masculinas con discapacidad versus el patrón dominante, que también influyen en la construcción conflictiva de ser hombre con discapacidad.

Sexo, sexualidad y discapacidad. Las personas con discapacidad han luchado durante mucho tiempo para tomar el control de sus cuerpos. Sin embargo, en la actualidad "experimentan represión sexual, poseen poca o ninguna autonomía sexual y deben tolerar las restricciones institucionales y legales sobre su conducta íntima" (Siebers 2012:38). Esta es una de las intersecciones en el desarrollo de los estudios críticos en discapacidad más potentes en la medida en que las manifestaciones de la sexualidad han tendido a ser tema tabú en sentido general, y han estado completamente invisibilizadas. De hecho, los estudios sociales de la discapacidad en sus inicios dedicaron poco tiempo a examinar esta relación. Como observamos con anterioridad, ha habido un desarrollo más significativo de la dimensión pública de la discapacidad que de la dimensión privada, emocional y que concierne a las experiencias de sujetos que no son colectivamente homogéneos. De la misma manera en que la reproducción es limitada y cercenada, desde los profesionales de la salud y la atención se controlan las manifestaciones de la sexualidad. "Rara vez se considera a las personas con discapacidad como sujetos deseantes u objetos de deseo. Y cuando el sexo y la discapacidad están vinculados [...] la conjunción suele ser la ocasión para la marginación o el asombro: la sexualidad de las personas con discapacidad se describe típicamente en términos de deficiencia trágica o exceso monstruoso. La lástima o el miedo [...] son las sensaciones más asociadas a la discapacidad" (McRuer y Mollow 2012:1).

Como se visualizó en el eje poder, producción de los cuerpos y opresión internalizada, la ideología de la capacidad constituye la base en torno a la cual giran las verdades sobre la discapacidad. Esta ideología también determina cómo pensamos la relación entre el sexo y la discapacidad. "La ausencia de habilidad o menor habilidad, según esta ideología, marca a una persona como menos humana. La preferencia por la capacidad impregna casi todos los valores de la cultura humana, incluida la capacidad para tener relaciones sexuales" (Siebers 2012: 41). 
En este sentido, resulta muy novedosa las discusiones teóricas que traen a la luz reflexiones relativas a la experiencia del sexo y la sexualidad en personas con discapacidad. Desde una perspectiva postfeminista, Kelly Fritsch narra lo que sucede en un encuentro íntimo cuando la persona con discapacidad recibe asistencia o ayuda para poder tener un intercambio sexual con su pareja: "Al preparar a alguien para el sexo (ayudarlo a bañarse o limpiarse, colocar su cuerpo, agregar lubricante, poner juguetes, correas o muebles al alcance de la mano) o ayudar a facilitar el acto (ayudar con el movimiento, buscar artículos según sea necesario, ayudar a desvestirse en momentos particulares), el asistente necesariamente se filtra y se desliza con los que están en la habitación. La persona con discapacidad expone las partes íntimas de su cuerpo y, al hacerlo, es vulnerable. Al permanecer con esta vulnerabilidad, el asistente no está completamente subsumido ni es capaz de mantener la distancia. Más bien, todos los involucrados están en un proceso continuo de devenir juntos a través de la circulación no cartografiada del deseo" (Fritsch 2010:9).

Se puede identificar así una variedad de identidades de género y sexos que tienen al menos dos características significativas. "Primero, representan la discapacidad no como un defecto que debe superarse para tener relaciones sexuales, sino como una encarnación compleja que mejora las actividades sexuales y el placer. En segundo lugar, dan a la sexualidad una dimensión política que redefine a las personas con discapacidad como ciudadanas sexuales. Es fundamental comprender que la ciudadanía sexual no se traduce simplemente en poder expresar la sexualidad en público, un cargo que siempre se ha impuesto a las minorías sexuales, sino en el derecho a liberarse del trato desigual de las sexualidades minoritarias y a crear nuevos modos de acceso para el sexo" (Siebers 2012:47).

Discapacidad racializada. La intersección raza y discapacidad ha sido desarrollada con mucha fuerza desde la teoría crítica de la raza y la discapacidad. Estos estudios abordan la discapacidad como una expresión de identidad interseccional en la que las características sociales devaluadas componen el estigma que resulta en la opresión de ciertos sujetos y "exploran las formas en las que tanto la raza como la capacidad se construyen socialmente y son interdependientes" (Connor, Ferri y Annamma 2016:27). Del mismo modo que los otros marcadores sociales, la raza constituye un dispositivo más para la dominación y subordinación. Las identidades múltiples y entrecruzadas de minorías raciales o étnicas y la discapacidad crean situaciones de marginación y opresión que difieren de las experiencias de las personas con discapacidad o de las minorías raciales propiamente tal. "La identificación, comprensión y explicación de las formas en que múltiples identidades o experiencias se combinan para crear barreras únicas resulta muy relevante" (Stienstra 2012:381).

Erevelles y Minear ilustran el valor de los enfoques interseccionales de la raza y la discapacidad dando cuenta de tres marcos de comprensión de las características constitutivas de las minorías identitarias: "marcos anticategóricos que insisten en la raza, la clase y el género como construcciones sociales; marcos intracategóricos que critican los enfoques meramente aditivos de las diferencias como estigmas estratificados; y marcos constitutivos que describen las condiciones estructurales dentro de las cuales las categorías sociales en los marcos anteriores se construyen (y se entrelazan) unas con otras en contextos históricos específicos" (Erevelles y Minear 2010:127). Ello deja pautado que las experiencias que viven algunos sujetos devienen de las formas en las que se les ha clasificado, por lo cual es un proceso completamente social y, por otro lado, evidencia que los marcadores sociales se filtran unos con otros y no pueden ser comprendidos como simples aditivos que se juntan en la situación social de un sujeto. Las imbricaciones de los marcadores 
determinan el reconocimiento específico de ese sujeto y las posibilidades a las que accederá en relación con el contexto específico donde desarrolla su vida. "Los individuos ubicados en las intersecciones de raza, clase, género y discapacidad se constituyen como no ciudadanos y no beneficiarios de las propias instituciones sociales (legales, educativas y de rehabilitación) que están diseñadas idealmente para protegerlos, nutrirlos y empoderarlos" (Erevelles y Minear 2010:127).

La teoría crítica de la raza y la discapacidad, como área emergente dentro de los estudios críticos de la raza, reconoce cómo los procesos de normalización del racismo y el capacitismo colocan a los cuerpos no deseados fuera de la categoría de lo normal para justificar su exclusión e incluso su terminación. "La única forma de resistir las narrativas dominantes que patologizan los cuerpos diferentes del ideal es proporcionar espacio para las narrativas contrarias de las personas oprimidas. Estas contra-narrativas no pueden sostenerse solas, sino que deben yuxtaponerse a las narrativas dominantes. Deben ser multidimensionales, considerando múltiples intersecciones de identidad" (Annamma, Jackson y Morrison 2017:153).

La potencia del enfoque interseccional para comprender procesos más complejos de opresión ha servido para visibilizar las prácticas propias del colonialismo y el neocolonialismo que subsisten en nuestros días. "Las etiquetas relacionadas con la discapacidad, como la debilidad y las enfermedades mentales, a menudo se consideraban sinónimos de cuerpos marcados opresivamente por la raza. Ante el temor de que tales características pudieran transmitirse de generación en generación y representar una amenaza adicional para la raza blanca dominante, las prácticas 'protectoras' como esterilizaciones forzadas, leyes rígidas de mestizaje, segregación residencial en guetos, barrios, reservas y otras instituciones estatales y, a veces incluso el genocidio se llevó a cabo en poblaciones no blancas bajo el disfraz protegido de la eugenesia" (Erevelles y Minear 2010:133).

\section{Ensambles posthumanos}

De acuerdo con Braidotti "lo humano es una convención normativa, no intrínsecamente negativa, pero con un elemento de poder reglamentario, y, por ende, instrumental a las prácticas de exclusión y discriminación. El estándar humano representa la regularidad, la regulación, y la reglamentación. Existe así un modelo generalizado de ser humano que es categóricamente distinto a los otros sexualizados, racializados, y con discapacidad. Este sujeto unitario propio del humanismo, a consideración de esta autora debe ser sustituido por uno más complejo y racional, caracterizado por la encarnación la sexualidad, la afectividad, la empatía y el deseo" (Braidotti 2015:38).

El posthumanismo es un campo no exento de polémicas por las discusiones y rupturas profundas que establece con formas tradicionales de comprensión de lo humano y "el hombre", sin embargo, se ha abierto un paso importante dentro de los estudios críticos de la discapacidad: "la discapacidad trae consigo conexiones interdependientes con otros seres humanos, tecnologías, entidades no humanas, corrientes de comunicación y redes de personas y no personas [...] la discapacidad es la condición posthumana por excelencia: porque exige nuevas ontologías, formas de relacionarse, vivir y morir. Los estudios posthumanos y los estudios críticos en discapacidad comparten una actitud contraria hacia los fundamentos ideológicos y normativos que se dan por sentado, de lo que significa ser un ciudadano valorado de la sociedad" (Goodley, Lawthom y Runswick-Cole 2014:348). Este este sentido, cuando se piensa la discapacidad desde este enfoque, se está considerando un espacio de comprensión que se encuentra fuera de las fronteras implantadas por la normalidad o el 
capacitismo. Este es un espacio revolucionario, donde nacen y coexisten formas no normativas, que contribuyen a consolidar el énfasis de los estudios críticos en considerar la discapacidad como variaciones de lo humano.

Uno de los postulados centrales del posthumanismo es restituir la idea de la "interdependencia con múltiples otros" (Goodley, Lawthom y Runswick-Cole 2014:348). Considera así al sujeto relacional como un elemento clave de la condición posthumana. Ello sin perder de vista que ha habido cambios relativamente significativos en las sociedades actuales que se descentran del yo individualizado como factor que define al sujeto, y consideran la importancia de la relación y la responsabilidad con otros. Desde los estudios feministas ha habido aportes importantes relacionados con la provisión de cuidados, por ejemplo, y también están otros casos como el humano y su perro guía, o el humano y la silla de ruedas o las prótesis biónicas y una discusión profunda en torno al concepto de "cyborg". Todas constituyen posibilidades transformadoras que ponen en tensión el binarismo en torno a la discapacidad y las concepciones dominantes de ser humano.

En este sentido, "el posthumanismo permite pensar en devenires múltiples, el sujeto con discapacidad se ubica en un conjunto de relaciones con muchos otros humanos y no humanos" (Goodley, Lawthom y Runswick-Cole 2014:353), que configuran de formas muy específicas y en diferentes grados su vida. Uno de los debates interesantes en torno a los ensamblajes diversos que postula este enfoque es el relativo a la aportación de Donna Haraway en torno al "cyborg" y cuál es su implicación para pensar la discapacidad. Si bien la materialidad del cyborg permitiría trascender el binarismo, en la medida en que ese ser humano se explica desde otro lugar, algunos autores han criticado los riesgos que tiene ocupar el concepto en la medida en que puede reproducir el binarismo entre cuerpo "normal" y cuerpo "anormal" que requiere la intromisión de tecnologías. Para Kafer "el término 'cyborg' en lugar de implicar una crítica de las categorías e ideologías existentes, se utiliza para perpetuar las distinciones [...] Las cualidades de los cyborg se convierten en marcadores de diferencia, lo que sugiere una diferencia esencial entre personas sin y con discapacidad. Cualquier tendencia transgresora potencial en el término se pierde cuando estas etiquetas se bloquean en ciertos cuerpos. El propio 'cyborg' se reifica, se reduce a un tipo particular de cuerpo" (Kafer 2013:110).

El uso del término es inútil si se quiere mirar la discapacidad desde un lente distinto al tradicional. Hace el juego a la necesidad de "normalizar" los cuerpos y cómo la tecnología se convierte en la herramienta más eficaz, pierde así todo el sentido crítico dado por Haraway destinado a trascender la centralidad del humano como yo individual. A consideración de Haraway: "El cyborg es una criatura en un mundo post genérico. No tiene relaciones con la bisexualidad, ni con la simbiosis preedípica, ni con el trabajo no alienado u otras seducciones propias de la totalidad orgánica, mediante una apropiación final de todos los poderes de las partes en favor de una unidad mayor" (Haraway 2020:2).

\section{Conclusiones}

Los estudios críticos en discapacidad constituyen un campo que ha ido consolidándose en las últimas tres décadas. Lo relevante de estos estudios es que permiten develar la variabilidad de situaciones sociales en las que la discapacidad cobra sentido porque ayudan a explicar la experiencia concreta de un sujeto que por múltiples intersecciones y producciones de verdad se encuentra excluido y 
oprimido, o porque el concepto hace sentido para comprender procesos reguladores más amplios que permiten al sistema social seguir operando desde las lógicas de un "sujeto normal".

A lo largo de este trabajo se proponen tres ejes de reflexión teórica: poder, producción de los cuerpos y opresión internalizada; interseccionalidad e identidades múltiples; y ensamblajes posthumanos. Los ejes resultan de una sistematización de los principales autores que producen en el área e intentan englobar, sin pretender agotar las múltiples aproximaciones e intersecciones en teorías, marcos más generales que se interconectan, dialogan, y producen en común conocimiento en torno a la discapacidad. Estos ejes muestran cómo las tendencias más actuales dentro de los estudios sociales sobre la discapacidad rompen con la propuesta que separa de manera radical la discapacidad (social) y la deficiencia (biológica), como si ambas no jugaran un rol importante en la traducción social del cuerpo de ciertos sujetos y en la producción del "sí mismo". Por otro lado, evidencian una focalización mucho mayor en el cuerpo, y como las interacciones de estos con ciertos contextos hacen posibles procesos opresivos más o menos explícitos.

Pensar la discapacidad implica así considerar las condiciones estructurales, económicas y políticas que oprimen y dificultan las posibilidades de desarrollo de un grupo de personas con características físicas y conductuales que son diferentes de la "norma" y, por otro lado, también considerar el carácter cultural, interseccional, flexible y variado en que se produce la experiencia de ser persona con discapacidad o de ser etiquetado con una discapacidad. Conceptos tales como biopoder, el estigma, el capacitismo y la opresión internalizada configuran sujetos específicos con discapacidad. Por otro lado, dar espacio teórico para comprender las situaciones únicas en las que se ubica un sujeto que es mujer, negra, con discapacidad severa en situación de pobreza, por ejemplo, resulta central. La mirada interseccional aporta a comprender que cuando se habla de personas con discapacidad, no se está haciendo alusión a un grupo homogéneo que tiene demandas comunes, sino que remite a un sinnúmero de experiencias que hacen que las demandas sean diferenciadas y que el concepto de discapacidad remita a múltiples lugares de enunciación. También, el tercer eje nos invita a superar definitivamente los postulados de la normalidad, del sujeto individual exitoso, por uno que interactúa, se relaciona, depende de otros sujetos humanos y no humanos. La persona con discapacidad podría considerarse como el posthumano por excelencia en la medida en que muchas de sus experiencias son compartidas y vividas desde uniones profundas y simbióticas con otros sujetos como puede ser un asistente o cuidador y/o con tecnologías con intromisiones varias en el cuerpo. La pregunta sobre qué es el hombre o el ser humano, el "sujeto normal", podría ser trascendida bajo esta lógica.

Resultó relevante reflexionar también sobre cómo estos ejes de discusión teórica permiten comprender la discapacidad como un concepto que es estratégico para explicar las formas en las que se producen a sí mismos y son producidos los sujetos en las sociedades actuales. Varios autores dan pista sobre la amplitud de este concepto que no solo refiere a los cuerpos con condiciones explícitas, sino también a un sinnúmero de procesos sociales a los que aplica la discapacidad. Como bien expresa Goodley, la discapacidad se convierte en un detonante importante "para pensar temas políticos y prácticas que interesan a todos" (Goodley 2013:632). De acuerdo con Erevelles podría tomarse como el principio organizador "en la construcción de categorías tales como el género, la raza, la clase o la orientación sexual” (Erevelles 1996:523). O podríamos considerar las palabras de Butler cuando se refiere al trastoque que produce pensar la discapacidad "de algunas cuestiones propias, que tienen que ver con nuestros valores [...] con cuestiones filosóficas del 'ser', del ser 
diferentes a nosotros, 'nosotros' y los 'otros' en la sociedad, en el mundo, en los lenguajes" (Butler 2013:1). Si se toma la discapacidad como aquel proceso social que justifica la generación de ciertas verdades dominantes que pueden excluir, dañar, limitar, o enajenar la vida de algunas personas, el concepto se convierte en algo potente.

\section{Bibliografía}

Abberley, P. 2008. El concepto de opresión y el desarrollo de una teoría social de la discapacidad. En L. Barton. Superar las barreras de la discapacidad, pp. 34-50. Ediciones Morata.

Annamma, S; Jackson, D; Morrison, D. 2017. Conceptualizing color-evasiveness: using dis/ability critical race theory to expand a color-blind racial ideology in education and society. Race Ethnicity and Education, 20(2), 147-162. https://doi.org/10.1080/13613324.2016.1248837

Braidotti, R. 2015. Lo posthumano. Gedisa.

Butler, J. 2007. El género en disputa: el feminismo y la subversión de la identidad. Paidós.

Butler, J. 2013. La discapacidad, una dificultad importante para toda la sociedad. X Congreso Argentino y V Latinoamericano de Educación Física y Ciencias. La Plata.

Campbell, F. 2009. Contours of ableism: the production of disability and abledness. Palgrave Macmillan.

Charlton, J. 2004. Nothing about us without us: disability oppression and empowerment. University of California Press.

Connor, D; Ferri, B; Annamma, S. 2016. Discrit. Disability studies and critical race theory in education. Teacher College Press.

Ellis, K; Garland-Thomson, R; Kent, M. 2019. Manifestos for the future of critical disability studies. Routledge.

Erevelles, N. 1996. Disability and the dialectics of difference. Disability \& Society, 11(4), 519-538. https://doi.org/10.1080/09687599627570

Erevelles, N; Minear, A. 2010. Unspeakable offenses: untangling race and disability in discourses of intersectionality. Journal of Literary \& Cultural Disability Studies, 4(2), 127-145. https://doi.org/10.3828/ilcds.2010.11

Foucault, M. 2007. Nacimiento de la biopolítica. Fondo de Cultura Económica.

Fritsch, K. 2010. Intimate assemblages: disability, intercorporeality, and the labour of attendant care. Critical Disability Discourses / Discours critiques dans le champ du handicap, 2, 1-14. https://cdd.journals.yorku.ca/index.php/cdd/article/view/23854

Garland-Thomson, R. 2017. Extraordinary bodies: figuring physical disability in American culture and literature. Columbia University Press.

Garland-Thomson, R. 2011. Misfits: A feminist materialist disability concept. Hypatia, 26(3), 591609. https://doi.org/10.1111/j.1527-2001.2011.01206.x

Goodley, D. 2013. Dis/entangling critical disability studies. Disability \& Society, 28(5), 631-644. https://doi.org/10.1080/09687599.2012.717884

Goodley, D; Lawthom, R. 2019. Critical disability studies, Brexit and Trump: a time of neoliberalableism. Rethinking History, 23(2), 233-251. https://doi.org/10.1080/13642529.2019.1607476 
Goodley, D; Lawthom, R; Runswick-Cole, K. 2014. Posthuman disability studies. Subjectivity, 7(4), 342-361. https://doi.org/10.1057/sub.2014.15

Goodley, D; Hughes, B; Davis, L. 2012. Disability and social theory. Palgrave Macmillan.

Haraway, D. 2020. Manifiesto cyborg. Kaotica libros.

Hughes, B. 2005. What can a Foucauldian analysis contribute to disability theory? In S. Tremain. Foucault and the government of disability, pp. 78-92. University of Michigan Press.

Kafer, A. 2013. Feminist, queer, crip. Indiana University Press.

Mallett, R; Runswick-Cole, K. 2015. Approaching disability: critical issues and perspectives. Routledge.

McRuer, R; Mollow, A. 2012. Sex and disability. Duke University Press.

Meekosha, H. 2006. What the hell are you? An intercategorical analysis of race, ethnicity, gender, and disability in the Australian body politic. Scandinavian Journal of Disability Research, 8(2-3), 161-176. https://doi.org/10.1080/15017410600831309

Meekosha, H; Shuttleworth, R. 2009. What's so 'critical' about critical disability studies? Australian Journal of Human Rights, 15(1), 47-75. https://doi.org/10.1080/1323238X.2009.11910861

Reeve, D. 2002. Negotiating psycho-emotional dimensions of disability and their influence on identity constructions. Disability \& Society, 17(5), 493-508. https://doi.org/10.1080/09687590220148487

Revuelta, B; Hernández, R. 2019. La teoría de Axel Honneth sobre justicia social, reconocimiento y experiencias del sujeto en las sociedades contemporáneas. Cinta de Moebio, 66, 333-346. https://doi.org/10.4067/s0717-554×2019000300333

Scior, K; Werner, S. 2019. Intellectual disability and stigma. Palgrave Macmillan.

Sheehan, R; Ali, A. 2019. Self-stigma in people with intellectual disabilities. In K. Scior, S. Werner. Intellectual disability and stigma, pp. 91-109. Palgrave Macmillan.

Shildrick, M. 2012. Critical disability studies. Rethinking the conventions for the age of postmodernity. In N. Watson; A. Roulstone; C. Thomas. Handbook of disability studies, pp. 30-41. Routledge.

Siebers, T. 2012. A sexual culture for disabled people. In R. McRuer, A. Mollow. Sex and disability, pp. 37-53. Duke University Press.

Stienstra, D. 2012. Race/ethnicity and disability studies. Towards an explicitly intersectional approach. In N. Watson, A. Roulstone, C. Thomas. Handbook of disability studies, pp. 376387. Routledge.

Thomas, C. 2004. Developing the social relational in the social model of disability: a theoretical agenda. In C. Barnes, G. Mercer. Implementing the social model of disability: theory and research, pp. 32-47. The Disability Press.

Tremain, S.L. 2020. Foucault and feminist philosophy of disability. University of Michigan Press.

Recibido el 4 Oct 2020

Aceptado el 26 Ene 2021 